\title{
Polyphenolic Components and Composition Differences of Fruit Polyphenols in Apple Landraces Grown in Cold Regions
}

\author{
Li Weiqun ${ }^{1,5^{*}}$, Zhao Jirong ${ }^{2 *}$, Wang Shuang ${ }^{1}$, Yu Wenquan ${ }^{3}$, Li Lin ${ }^{1}$, Bu Haidong ${ }^{3}$, Wang Kun ${ }^{4}$, Liu Chang ${ }^{3}{ }^{-1}$ \\ 1 Heilongjiang Academy of Agricultural Sciences Institute of Soil Fertility and Environmental Resources, Heilongjiang Key Laboratory of Soil Environment \\ and Plant Nutrition, Harbin, 150086, China \\ 2 College of Life Science Yan'an University; Shanxi Key Laboratory of Chinese Jujube, Yan'an, 716000, China \\ 3 Mudanjiang Branch of Heilongjiang Academy of Agricultural Sciences, Heilongjiang Provincial Key Laboratory of Fruit Trees, Mudanjiang, 157000, China \\ 4 Research Institute of Pomology OF CAAS, Key Laboratory of Biology and Genetic Improvement of Horticultural Crops (Germplasm Resources Utilization) \\ Ministry of Agriculture, Xingcheng, 125100, China \\ 5 Agricultural Research Institute of the $10^{\text {th }}$ Division of Xinjiang Production and Construction Corps, Beitun, 836000, China \\ * These authors contributed equally to this work \\ $\nabla$ Corresponding email: changchang_3000@163.com
}

International Journal of Horticulture, 2021, Vol.11, No.4 doi: 10.5376/ijh.2021.11.0004

Received: 24 Sep., 2021

Accepted: 30 Sep., 2021

Published: 15 Oct., 2021

Copyright $\odot 2021$ Li et al., This article was first published in Molecular Plant Breeding in Chinese, and here was authorized to translate and publish the paper in English under the terms of Creative Commons Attribution License, which permits unrestricted use, distribution, and reproduction in any medium, provided the original work is properly cited.

Preferred citation for this article:

Li W.Q., Zhao J.R., Wang S., Yu W.Q., Li L., Bu H.D., Wang K., and Liu C. 2021, Polyphenolic components and composition differences of fruit polyphenols in apple landraces grown in cold regions, International Journal of Horticulture, 11(4): 1-10 (doi: 10.5376/ijh.2021.11.0004)

\begin{abstract}
The composition and content of polyphenols in fruit pericarp and pulp of 12 Apple landraces grown of Heilongjiang Province were determined by ultra-high performance liquid chromatography (UPLC), and the differences in the composition and content characteristics of polyphenols in different varieties were studied, and the main factor analysis and cluster analysis were carried out. The results showed that 5 kinds of 20 kinds of polyphenols were detected in 12 Apple landraces, the main phenolic substances in pericarp were epicatechin and catechin, and the main phenolic substances in pulp were chlorogenic acid, catechin and procyanidin. There were significant differences in the composition and content of polyphenols among different varieties, the smallest coefficient of variation was quercetin 3-glucoside (37.48\%), and the largest coefficient of variation was rutin (267.18\%). It was found that the content of proanthocyanidins in pericarp and pulp was higher by principal component analysis. Based on the content of polyphenol components, it was found that the results of pulp clustering were more consistent with the field phenotypic identification results and kinship. The results provide data support and theoretical basis for processing and utilization of phenolic substances and breeding of local varieties in Heilongjiang province.
\end{abstract}

Keywords Heilongjian; Apples; Fruits; Polyphenols; Principal component analysis

Heilongjiang Province, located in the northeast of China, has a temperate continental monsoon climate and is the province with the lowest average temperature in China. The average temperature in summer is $20^{\circ} \mathrm{C} \sim 25^{\circ} \mathrm{C}$, the average temperature in winter is $-15^{\circ} \mathrm{C} \sim-30^{\circ} \mathrm{C}$, the annual average temperature in the whole province is $-5^{\circ} \mathrm{C} \sim 5^{\circ} \mathrm{C}$, and the average temperature of seven months is below $0^{\circ} \mathrm{C}$ throughout the year. The temperature changes greatly, which is very conducive to nutrient accumulation. At the same time, due to the high nutrient content of the black soil in the northeast, the fruits are of good quality, which has unique conditions for the development of fruit trees (Guo, 2012). Due to low temperatures, short growing seasons and severe winters, apples in the main producing areas cannot be grown in Heilongjiang. Over the past 60 years, Heilongjiang Province has bred a number of apple varieties with good fruit shape, high quality, excellent storage capacity and strong cold resistance through introduction, selection, breeding and inspection, but the specific components of these varieties are still unclear.

Polyphenols are one of the important secondary metabolites in plants, mainly found in skin, root, leaf and fruit (Shi and Di, 2000, Science Press, pp. 9-12), which are different under different growth environments and storage modes (Van et al., 2001). A large number of studies have shown that phenolic substances are closely related to many aspects of fruit quality. Cornflower pigments determine the redness of apple, flavonoids determine the yellowness, and dihydrochalcones affect the sweetness of apple (Feng et al., 2008; Han et al., 2019). Polyphenols have strong antioxidant capacity. It is reported that their antioxidant capacity and various biological activities are 100 times higher than those of tea polyphenols ( $\mathrm{Lu}$ and Foo, 2000). It can effectively inhibit superoxide anion free 
radicals produced by lipid peroxidation (Liu et al., 2005), the ability of which can reach 10 30 times that of VC and VE (Wang et al., 2011). The scavenging rate of polyphenols on lipid peroxidation free radicals is above $90 \%$ (Li et al., 2010). It is widely used in functional drinks, food, cosmetics and medical treatment. It is of great significance to study the phenolic components of fruits during the ripening period for the development of landraces in Heilongjiang Province.

At present, the research on apple polyphenols is mainly focused on the classification, components, content, antioxidant capacity, extraction methods and differences of wild resources, main varieties and local varieties (Song et al., 2006; Zhang et al., 2015; Wang et al., 2017a; Zhao, 2019). But there is less research on apple polyphenol pulp in China (Zhang et al., 1999; Zhao et al., 2015; Yan et al., 2018), and there is no report on the analysis of the composition and content of phenolic substances in the pericarp and pulp of local apple varieties. In this study, 12 apple landraces grown in Heilongjiang Province were used as test materials, and the components and content of pericarp and pulp were deeply studied and analyzed by high performance liquid chromatography, so as to reveal the differential characteristics of polyphenolic content in apple fruits during the ripening period in cold regions, and provide data support and theoretical basis for the processing and utilization of phenolic substances and the further breeding of apple varieties.

\section{Results and Analysis}

\subsection{Components and content of different components in pericarp of apple landraces grown in Heilongjiang Province}

A total of 5 categories and 20 kinds of phenolic substances were detected in pericarp of 12 apple landraces grown in Heilongjiang Province (Table 1), including 7 kinds of hydroxycinnamic acids, 4 kinds of flavonals, 1 kind of dihydrochalcone, 3 kinds of procyanidins and 5 kinds of procyanidins. The pericarp of 'Cuiping' contains 18 kinds of phenolic substances. The pericarp of 'Huahong', 'Number 1 Jixi' and 'Zikui' contains 16 kinds of phenolic substances. The pericarp of '124' contains 15 kinds of phenolic substances. The pericarp of 'Longhong' contains 14 kinds of phenolic substances. The pericarp of 'Longshuai' contains 13 kinds of phenolic substances. The pericarp of 'Tianfeng' contains 12 kinds of phenolic substances. The pericarp of 'Qizaohong', 'Shuangqiu' and 'Zixiang' contains 11 kinds of phenolic substances. The pericarp of 'Jixinguo' contains 8 kinds of phenolic substances. The content of polyphenols in pericarp varied significantly among different varieties. The lowest coefficient of variation was quercetin 3-glucoside (37.48\%), and the highest coefficient of variation was rutin (267.18\%). Gallic acid was only detected in 'Cuiping', protocatechuic acid was only detected in 'Huahong', kaempferol was only detected in 'Qizaohong' and '124', and neochlorogenic acid was only detected in 'Cuiping', 'Number 1 Jixi' and '124'.

\subsection{Components and content of different components in pulp of apple landraces grown in Heilongjiang Province}

A total of 5 categories and 16 kinds of phenolic substances were detected in pulp of 12 apple landraces grown in Heilongiiang Province (Table 2), including 3 kinds of hydroxycinnamic acids, 5 kinds of flavonals, 1 kind of dihydrochalcone, 2 kinds of procyanidins and 5 kinds of procyanidins. The content of polyphenols in pulp of different varieties was significantly different, and the coefficient of variation was $60.34 \% \sim 217.07 \%$. The highest coefficient of variation was catechins and the lowest coefficient of variation was quercetin. Gallic acid, protocatechuic acid, neochlorogenic acid and cyanidin-3-O-glucoside were not detected in the pulp of 12 varieties. Cyanidin-3-galactoside was only detected in 'Zixiang', and anthocyanin rhamnoside was only detected in 'Qizaohong'. 
International Journal of Horticulture, 2021, Vol.11, No.4, 1-10

http://hortherbpublisher.com/index.php/ijh

Table1 Composition and content of polyphenols in pericarp of 12 apple landraces grown in Heilongjiang Province mg $\cdot \mathrm{kg}-1$ (FW)

\begin{tabular}{|c|c|c|c|c|c|c|c|c|c|c|c|}
\hline \multirow[t]{2}{*}{ Variety } & \multicolumn{7}{|c|}{$\underline{\text { Hydroxycinnamic acids }}$} & \multicolumn{4}{|l|}{ Flavonols } \\
\hline & GALAC & PROAC & CINAC & CHLAC & NEOAC & CRYAC & KAE & QUERU & QUEAL & QUEGL & QUERC \\
\hline 'Longshuai' & nd & nd & $7.80 \pm 0.02$ & $10.40 \pm 0.26$ & nd & $4.03 \pm 0.44$ & nd & $5.72 \pm 0.01$ & $16.93 \pm 0.98$ & $7.83 \pm 0.59$ & $17.99 \pm 2.06$ \\
\hline ‘Huahong’' & nd & $5.11 \pm 0.00$ & $7.87 \pm 0.21$ & $29.44 \pm 0.01$ & nd & $4.20 \pm 0.63$ & nd & $6.68 \pm 0.00$ & $69.36 \pm 0.00$ & $13.47 \pm 0.00$ & $16.01 \pm 1.97$ \\
\hline 'Cuiping' & $16.57 \pm 0.01$ & nd & $8.20 \pm 0.71$ & $12.45 \pm 1.09$ & $4.14 \pm 0.13$ & $4.89 \pm 1.18$ & nd & $7.40 \pm 0.57$ & $23.53 \pm 1.98$ & $12.08 \pm 0.81$ & $20.19 \pm 1.24$ \\
\hline 'Jixinguo' & nd & nd & nd & $3.69 \pm 0.39$ & nd & nd & nd & nd & $6.22 \pm 0.01$ & $7.54 \pm 0.00$ & $14.13 \pm 1.02$ \\
\hline ‘Qizaohong' & nd & nd & $7.80 \pm 0.10$ & $3.68 \pm 0.02$ & nd & $4.41 \pm 0.00$ & $9.50 \pm 0.39$ & nd & $5.36 \pm 0.00$ & $7.46 \pm 0.18$ & $14.34 \pm 1.03$ \\
\hline 'Number 1 Jixi' & nd & nd & $7.78 \pm 0.00$ & $3.23 \pm 0.13$ & $4.36 \pm 0.00$ & $4.88 \pm 2.38$ & nd & nd & $4.93 \pm 0.02$ & $22.36 \pm 0.00$ & $11.73 \pm 1.48$ \\
\hline 'Shuangqiu' & nd & nd & nd & $3.32 \pm 0.19$ & nd & nd & nd & nd & nd & $8.15 \pm 0.71$ & $12.08 \pm 2.10$ \\
\hline 'Zikui' & nd & nd & $7.67 \pm 0.00$ & $47.12 \pm 1.35$ & nd & $5.12 \pm 0.67$ & nd & $6.47 \pm 0.00$ & $6.56 \pm 1.21$ & $12.49 \pm 1.64$ & $11.82 \pm 1.31$ \\
\hline '124' & nd & nd & $9.09 \pm 1.44$ & $3.71 \pm 0.37$ & $4.16 \pm 0.00$ & $3.66 \pm 0.07$ & $11.00 \pm 0.39$ & $119.11 \pm 0.67$ & $16.59 \pm 0.00$ & $19.37 \pm 2.15$ & nd \\
\hline 'Longhong' & nd & nd & $7.96 \pm 0.25$ & $14.68 \pm 2.34$ & nd & $6.42 \pm 2.07$ & nd & $5.95 \pm 0.01$ & $88.38 \pm 2.62$ & $15.62 \pm 1.75$ & $49.64 \pm 1.19$ \\
\hline 'Zixiang' & nd & nd & $8.05 \pm 0.36$ & $3.16 \pm 0.19$ & nd & nd & nd & nd & $14.84 \pm 0.00$ & $13.43 \pm 1.21$ & $25.36 \pm 2.82$ \\
\hline 'Tianfeng' & nd & nd & $12.82 \pm 0.00$ & $3.06 \pm 0.16$ & nd & $3.66 \pm 0.00$ & nd & nd & $10.44 \pm 1.58$ & $11.91 \pm 0.98$ & nd \\
\hline $\begin{array}{l}\text { Mutations } \\
\text { coefficient } / \%\end{array}$ & & & 50.83 & 119.10 & 180.97 & 64.03 & 234.30 & 267.18 & 126.25 & 37.48 & 79.64 \\
\hline \multirow[t]{2}{*}{ Variety } & \multicolumn{2}{|c|}{ Dihydrochalcones } & \multicolumn{3}{|c|}{ Anthocyanins } & \multicolumn{6}{|c|}{ Proanthocyanidins } \\
\hline & PHL & & CYAGA & CAYGL & ANTRH & PROB1 & & CAT & PROB4 & PROB2 & EP \\
\hline 'Longshuai' & $16.02 \pm 1.67$ & & $9.85 \pm 1.28$ & nd & nd & $3.04+0.02$ & & $2.89 \pm 2.09$ & nd & $8.46 \pm 0.45$ & $32.78 \pm 1.61$ \\
\hline 'Huahong' & $16.94 \pm 2.67$ & & $58.35 \pm 0.00$ & $6.63 \pm 0.01$ & $105.64 \pm 0.01$ & $13.01 \pm 2.08$ & & $94.61 \pm 0.00$ & $46.86 \pm 0.01$ & nd & $19.49 \pm 0.55$ \\
\hline 'Cuiping' & $77.41 \pm 1.58$ & & $12.30 \pm 1.83$ & $6.03 \pm 0.00$ & $6.25 \pm 0.00$ & $7.79 \pm 1.11$ & & $15.65 \pm 1.37$ & $2.65 \pm 0.00$ & $20.74 \pm 1.55$ & $22.89 \pm 2.55$ \\
\hline 'Jixinguo' & nd & & nd & nd & $12.88 \pm 1.03$ & nd & & nd & $9.29 \pm 0.00$ & $6.71 \pm 2.71$ & $10.20 \pm 0.66$ \\
\hline 'Qizaohong' & nd & & nd & $16.07 \pm 0.20$ & nd & nd & & $5.35 \pm 1.16$ & $2.49 \pm 0.00$ & nd & $73.77 \pm 2.02$ \\
\hline 'Number 1 Jixi' & $8.56 \pm 0.00$ & & $33.04 \pm 0.00$ & $14.15 \pm 1.96$ & $11.28 \pm 0.00$ & $3.01 \pm 0.99$ & & $5.88 \pm 1.36$ & $3.49 \pm 1.02$ & $7.12 \pm 0.89$ & $8.81 \pm 0.44$ \\
\hline 'Shuangqiu' & $7.75 \pm 0.00$ & & nd & $6.97 \pm 0.00$ & $6.88 \pm 0.03$ & $4.04 \pm 2.12$ & & $0.07 \pm 0.00$ & $2.89 \pm 0.11$ & $5.06 \pm 0.20$ & $8.07 \pm 0.00$ \\
\hline 'Zikui' & $18.06 \pm 1.89$ & & $28.87 \pm 0.00$ & $5.80 \pm 0.00$ & $16.08 \pm 1.44$ & $5.76 \pm 1.95$ & & $37.93 \pm 0.00$ & $7.45 \pm 1.56$ & $7.42 \pm 0.79$ & $22.71 \pm 0.00$ \\
\hline '124' & $8.31 \pm 0.29$ & & $15.56 \pm 0.00$ & $5.78 \pm 0.00$ & nd & nd & & $107.59 \pm 0.37$ & $3.51 \pm 0.86$ & $5.13 \pm 0.00$ & $28.07 \pm 1.29$ \\
\hline 'Longhong' & $41.53 \pm 1.07$ & & $13.31 \pm 2.41$ & $5.69 \pm 0.00$ & nd & $7.39 \pm 1.47$ & & $7.71 \pm 2.68$ & nd & $14.35 \pm 1.38$ & $24.79 \pm 1.82$ \\
\hline 'Zixiang' & $8.16 \pm 0.48$ & & nd & nd & $9.38 \pm 1.29$ & $3.04 \pm 0.58$ & & $1.01 \pm 0.01$ & nd & $5.76 \pm 0.63$ & $19.02 \pm 1.22$ \\
\hline 'Tianfeng' & $50.51 \pm 0.01$ & & nd & $9.80 \pm 0.00$ & nd & $11.23 \pm 0.00$ & & $2.38 \pm 1.57$ & $7.38 \pm 0.00$ & $5.49 \pm 0.07$ & $8.65 \pm 0.03$ \\
\hline $\begin{array}{l}\text { Mutations } \\
\text { coefficient } / \%\end{array}$ & 111.24 & & 125.91 & 80.07 & 209.68 & 88.71 & & 161.69 & 159.72 & 78.92 & 76.91 \\
\hline
\end{tabular}

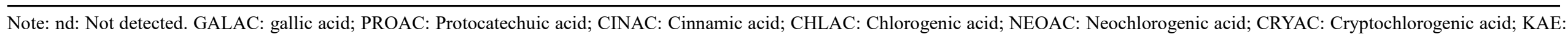

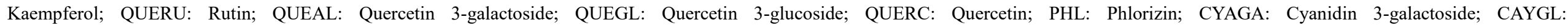
Cyanidin-3-O-glucoside; ANTRH: Anthocyanin rhamnoside; PROB1: Proanthocyanidin B1; PROB2: Proanthocyanidin B2; PROB4: Proanthocyanidin B4; CAT: Catechins; EP: Epicatechin 
International Journal of Horticulture, 2021, Vol.11, No.4, 1-10

http://hortherbpublisher.com/index.php/ijh

Table 2 Composition and content of polyphenols in pulp of 12 apple landraces grown in Heilongjiang Province mg $\cdot \mathrm{kg}-1(\mathrm{FW})$

\begin{tabular}{|c|c|c|c|c|c|c|c|c|c|c|c|}
\hline \multirow[t]{2}{*}{ Variety } & \multicolumn{7}{|c|}{ Hydroxycinnamic acids } & \multicolumn{4}{|l|}{ Flavonols } \\
\hline & GALAC & PROAC & CINAC & CHLAC & NEOAC & CRYAC & KAE & QUERU & QUEAL & QUEGL & QUERC \\
\hline 'Longshuai' & nd & nd & $7.64 \pm 0.00$ & $14.77 \pm 1.18$ & nd & $4.22 \pm 0.83$ & nd & nd & $4.98 \pm 0.00$ & nd & $10.36 \pm 0.55$ \\
\hline 'Huahong' & nd & nd & nd & $48.24 \pm 2.51$ & nd & $4.08 \pm 0.47$ & nd & nd & nd & $7.3 \pm 0.06$ & $10.58 \pm 0.34$ \\
\hline ‘Cuiping’' & nd & nd & nd & $2.94 \pm 0.03$ & nd & $10.90 \pm 0.00$ & nd & nd & nd & nd & $10.10 \pm 0.00$ \\
\hline 'Jixinguo' & nd & nd & nd & $8.29 \pm 0.00$ & nd & $17.81 \pm 1.82$ & nd & nd & nd & nd & $10.25 \pm 0.24$ \\
\hline 'Qizaohong' & nd & nd & $7.59 \pm 0.00$ & $3.74 \pm 0.28$ & nd & $3.71 \pm 0.00$ & nd & nd & nd & nd & nd \\
\hline 'Number 1 Jixi' & nd & nd & $7.78 \pm 0.27$ & $3.20 \pm 0.17$ & nd & $3.56 \pm 0.10$ & $9.97 \pm 0.00$ & $5.90 \pm 0.03$ & $5.15 \pm 0.20$ & $7.29 \pm 0.03$ & nd \\
\hline 'Shuangqiu' & nd & nd & nd & $29.62 \pm 1.57$ & nd & $3.88 \pm 0.17$ & nd & nd & nd & $7.28 \pm 0.00$ & $10.09 \pm 0.01$ \\
\hline 'Zikui' & nd & nd & nd & nd & nd & $42.68 \pm 2.83$ & nd & nd & $5.14 \pm 0.29$ & $7.18 \pm 0.00$ & $10.66 \pm 0.10$ \\
\hline '124’' & nd & nd & nd & $13.59 \pm 0.00$ & nd & $3.39 \pm 0.54$ & nd & nd & $5.01 \pm 0.01$ & $7.11 \pm 0.00$ & $10.39 \pm 0.07$ \\
\hline 'Longhong' & nd & nd & $7.59 \pm 0.01$ & $4.12 \pm 0.10$ & nd & $3.56 \pm 0.08$ & $8.98 \pm 0.08$ & $7.08 \pm 0.00$ & $5.23 \pm 0.00$ & $7.65 \pm 0.00$ & nd \\
\hline 'Zixiang' & nd & nd & $7.58 \pm 0.00$ & $47.35 \pm 0.00$ & nd & $4.61 \pm 0.62$ & nd & nd & $5.09 \pm 0.00$ & $7.24 \pm 0.00$ & $10.27 \pm 0.19$ \\
\hline 'Tianfeng' & nd & nd & $7.60 \pm 0.02$ & $4.09 \pm 0.90$ & nd & $3.46 \pm 0.48$ & $8.98 \pm 0.51$ & $5.78 \pm 0.00$ & $5.55 \pm 0.87$ & $7.11 \pm 0.00$ & $10.32 \pm 0.32$ \\
\hline $\begin{array}{l}\text { Mutations } \\
\text { coefficient } / \%\end{array}$ & & & 104.46 & 115.26 & & 130.46 & 181.21 & 181.96 & 88.39 & 73.91 & 60.34 \\
\hline \multirow[t]{2}{*}{ Variety } & \multicolumn{2}{|c|}{ Dihydrochalcones } & Anthocyar & & & \multicolumn{6}{|c|}{ Proanthocyanidins } \\
\hline & \multicolumn{2}{|c|}{ PHL } & CYAGA & CAYGL & ANTRH & \multicolumn{2}{|c|}{ PROB1 } & CAT & PROB4 & PROB2 & EP \\
\hline 'Longshuai' & \multicolumn{2}{|l|}{$11.48 \pm 0.00$} & nd & nd & nd & \multicolumn{2}{|l|}{$5.29 \pm 1.98$} & nd & $12.26 \pm 0.00$ & $8.47 \pm 1.54$ & $20.35 \pm 0.00$ \\
\hline ‘Huahong' & \multicolumn{2}{|l|}{ nd } & nd & nd & nd & \multicolumn{2}{|l|}{$11.54 \pm 0.61$} & nd & $2.58 \pm 0.00$ & $37.95 \pm 2.57$ & $8.05 \pm 0.00$ \\
\hline ‘Cuiping’' & \multicolumn{2}{|l|}{$7.86 \pm 0.00$} & nd & nd & nd & \multicolumn{2}{|l|}{$2.49 \pm 0.00$} & $0.06 \pm 0.00$ & nd & $8.95 \pm 1.77$ & nd \\
\hline 'Jixinguo' & \multicolumn{2}{|l|}{ nd } & nd & nd & nd & \multicolumn{2}{|l|}{$3.36 \pm 0.01$} & nd & $2.76 \pm 0.00$ & $15.01 \pm 1.29$ & $10.68 \pm 0.12$ \\
\hline 'Qizaohong' & \multicolumn{2}{|l|}{ nd } & nd & nd & $6.14 \pm 0.00$ & \multicolumn{2}{|l|}{$15.30 \pm 2.54$} & $129.51 \pm 0.00$ & $2.99 \pm 0.00$ & $5.85 \pm 0.52$ & $21.29 \pm 0.00$ \\
\hline 'Number 1 Jixi' & \multicolumn{2}{|l|}{ nd } & nd & nd & nd & \multicolumn{2}{|l|}{$24.84 \pm 2.41$} & $10.85 \pm 0.00$ & nd & $5.67 \pm 0.34$ & $7.75 \pm 0.00$ \\
\hline ‘Shuangqiu' & \multicolumn{2}{|l|}{ nd } & nd & nd & nd & \multicolumn{2}{|l|}{$4.83 \pm 0.64$} & nd & $2.62 \pm 0.05$ & $26.75 \pm 0.93$ & nd \\
\hline ‘Zikui’' & \multicolumn{2}{|l|}{$14.19 \pm 1.27$} & nd & nd & nd & \multicolumn{2}{|l|}{$4.53 \pm 0.00$} & nd & $2.59 \pm 0.00$ & $5.18 \pm 0.00$ & $9.15 \pm 0.66$ \\
\hline ‘124’ & \multicolumn{2}{|l|}{$10.31 \pm 0.82$} & nd & nd & nd & \multicolumn{2}{|l|}{$2.63 \pm 0.00$} & nd & $2.54 \pm 0.00$ & $5.76 \pm 0.10$ & $10.54 \pm 0.19$ \\
\hline 'Longhong' & \multicolumn{2}{|l|}{ nd } & nd & nd & nd & \multicolumn{2}{|l|}{$2.57 \pm 0.27$} & $117.69 \pm 2.39$ & $2.81 \pm 0.11$ & nd & $23.53 \pm 2.65$ \\
\hline 'Zixiang' & $11.87 \pm 0.00$ & & $7.59 \pm 0.00$ & nd & nd & $2.67 \pm 0.00$ & & nd & $2.56 \pm 0.00$ & $7.68 \pm 2.09$ & $31.36 \pm 0.00$ \\
\hline 'Tianfeng' & $16.95 \pm 0.90$ & & nd & nd & nd & $3.04 \pm 0.00$ & & $5.36 \pm 0.00$ & nd & $5.86 \pm 1.07$ & $13.60 \pm 0.70$ \\
\hline $\begin{array}{l}\text { Mutations } \\
\text { coefficient/\% }\end{array}$ & 110.17 & & & & & 100.07 & & 217.07 & 114.25 & 97.07 & 72.74 \\
\hline
\end{tabular}

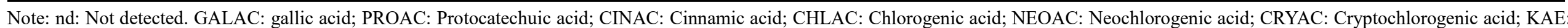

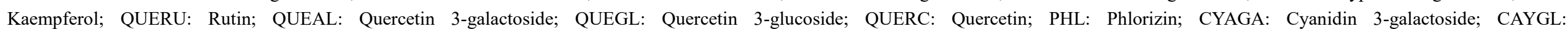

Cyanidin-3-O-glucoside; ANTRH: Anthocyanin rhamnoside; PROB1: Proanthocyanidin B1; PROB2: Proanthocyanidin B2; PROB4: Proanthocyanidin B4; CAT: Catechins; EP: Epicatechin 


\subsection{Principal component analysis of polyphenolic components in fruits of apple landraces grown in Heilongjiang Province}

In order to more intuitively analyze the polyphenol composition of 12 apple landraces, the principal component analysis of polyphenolic content was carried out. The cumulative contribution rate of the first four principal components of relative content of pericarp polyphenols was $74.35 \%$. The contribution rate of the first principal component was $27.5 \%$ and played a major role, mainly represented by hydroxycinnamic acids, anthocyanins and proanthocyanidins; The contribution rate of the second principal component was $18.98 \%$, mainly represented by hydroxycinnamic acids, dihydrochalcones and proanthocyanidins; The contribution rate of the third principal component was $17.91 \%$, mainly represented by flavonols and proanthocyanidins; The contribution rate of the fourth principal component is $9.96 \%$, mainly represented by hydroxycinnamic acids and anthocyanins.

Take the first principal component as the abscissa and the second principal component and the third principal component as the ordinate to draw the scatter diagrams to show the distribution of apple landraces (Figure 1). The higher the principal component was, the higher the relative content was. The first principal component of 'Huahong' and 'Zikui' was high, the second principal component of 'Cuiping' was high, and the third principal component of 'Huahong' and '124' was high. Considering the distribution of all three principal components, the principal factor of 'Huahong' was with positive value and high score, which had the highest cumulative value of various polyphenolic components. At the same time, it was found that rutin, quercetin-glucoside, quercetin and cyanidin-3-glucoside were distributed near the origin of the second and third principal components, indicating that the content of flavonols was very low, and other components were scattered in the two scatter diagrams, indicating that the content of polyphenols was very rich, which was basically consistent with the data in Table 1.
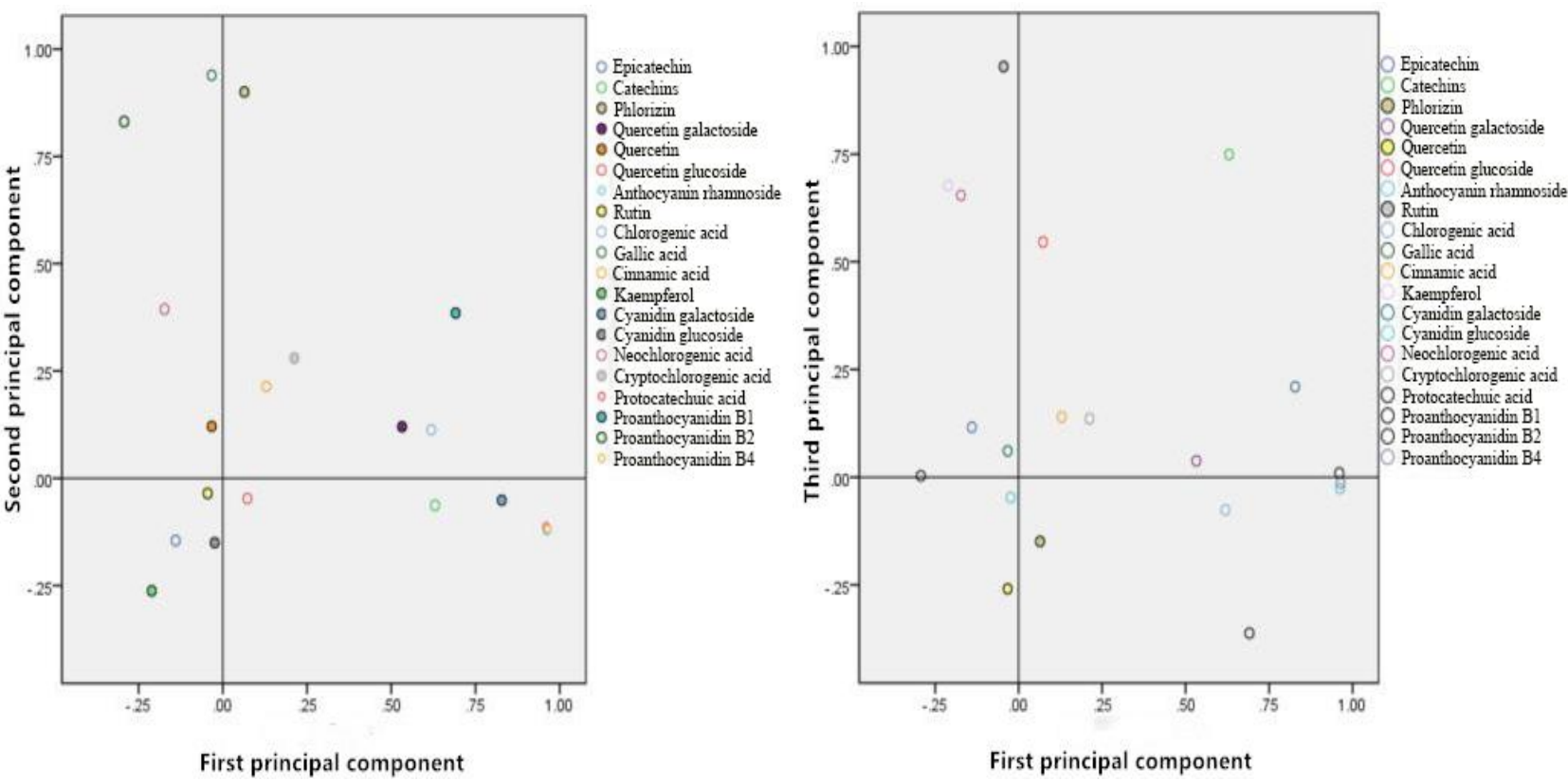

Figure 1 Principal component analysis of relative content of pericarp polyphenols in 12 apple landraces

The cumulative contribution rate of the first four principal components of relative content of pulp polyphenols was $81.51 \%$. The contribution rate of the first principal component was $33.44 \%$ and played a major role, mainly represented by flavonols, dihydrochalcones and proanthocyanidins; The contribution rate of the second principal component was $19.55 \%$, mainly represented by phenolic acids and flavonols; The contribution rate of the third principal component was $15.20 \%$, mainly represented by phenolic acids and proanthocyanidins; The contribution rate of the fourth principal component is $13.33 \%$, mainly represented by chlorogenic acid substances of phenolic acids and proanthocyanidins B2 substances of proanthocyanidins. 
Take the first principal component of pulp as the abscissa and the second principal component and the third principal component as the ordinate to draw the scatter diagrams (Figure 2). The distribution showed that the higher the principal component was, the higher the relative content was. The first principal component of quercetin and phlorizin distributed in the positive electrode and proanthocyanidins B1 and catechins distributed in the negative electrode was high. The second principal component of kaempferol, rutin, quercetin galactoside and quercetin glucoside was high. The third principal component of cinnamic acid, proanthocyanidins B4 and epicatechin was high. Considering the characteristics of the three principal components and the relative content, it was found that 'Qizaohong' was the highest, followed by 'Longhong', which was basically consistent with the data in Table 2.
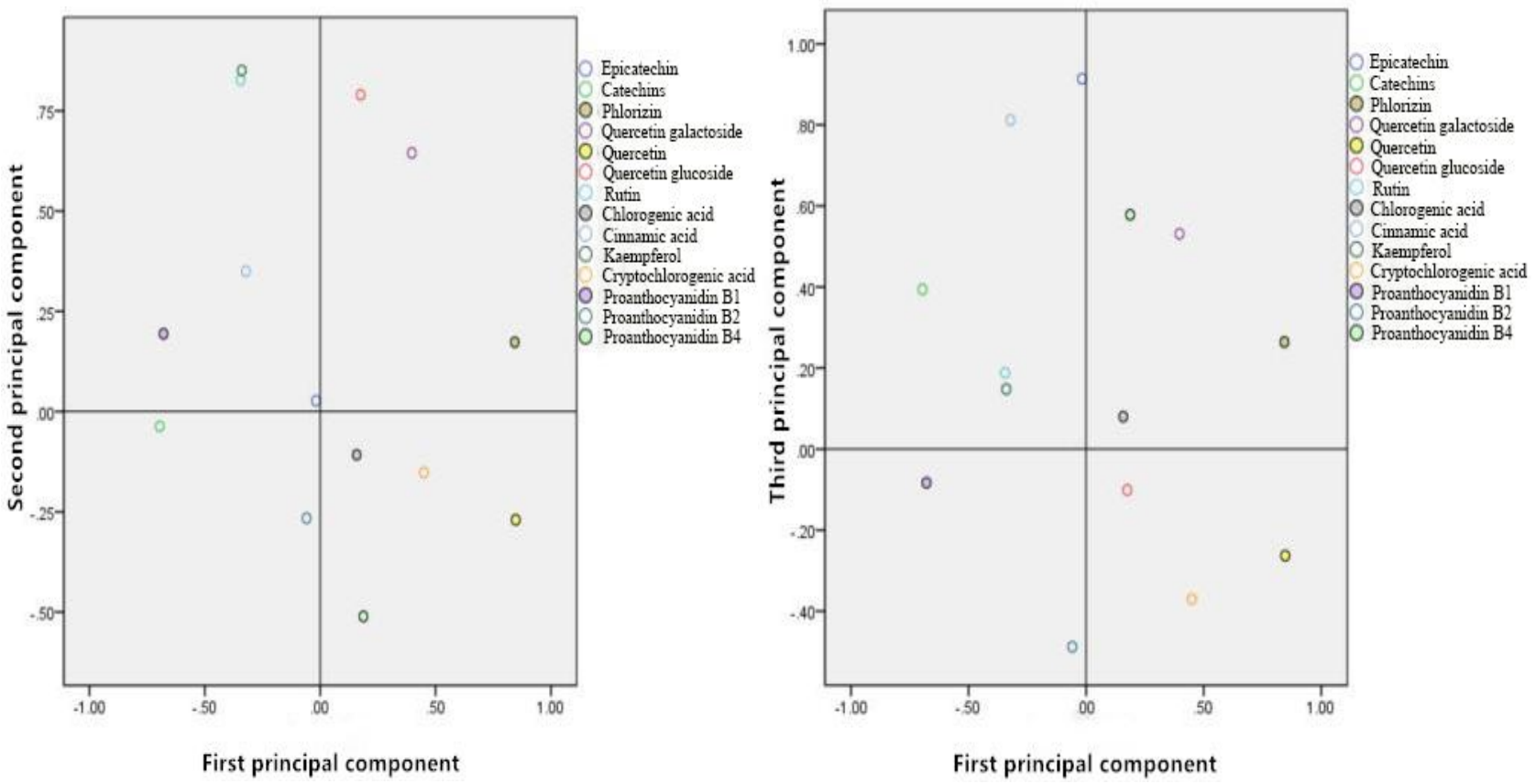

Figure 2 Principal component analysis of relative content of pulp polyphenols in 12 apple landraces

\subsection{Cluster analysis of polyphenols of apple landraces grown in Heilongjiang Province}

Taking the relative content of polyphenols in pericarp and pulp as variables, cluster analysis was carried out on 12 apple landraces grown in Heilongjiang Province. By clustering the pericarp (Figure 3) and pulp (Figure 4), they were both divided into two categories, but they were not exactly the same. It can be seen from Figure 3 that 'Jixinguo' and 'Shuangqiu' were first clustered into one group, 'Longshuai' and 'Zixiang' were clustered into one group, and then these two groups were grouped into one group. 'Qizaohong' and 'Zikui' were clustered into one group, '124' and 'Longhong' were clustered into one group, and then they were clustered together with 'Tianfeng', 'Number 1 Jixi', 'Cuiping' and the other two groups. We can see the resource diversity of apple landraces. Due to high content of anthocyanins, 'Huahong' was clustered into one group alone. It can be seen from Figure 4 that the contents of 'Qizaohong' and 'Longhong' were similar, so they were first clustered into one group, and the second group includes 'Huahong', 'Zixiang' and the other varieties. 'Jixinguo' and 'Shuangqiu' were clustered into one group, 'Longshuai' and 'Number 1 Jixi' were clustered into one group, '124' and 'Tianfeng' were clustered into one group, and then these three groups were clustered together with 'Cuiping' and 'Zikui', and were clustered into a big group with 'Huahong' and 'Zixiang'. The content in pulp of 'Huahong' was not the highest, and 'Qizaohong' and 'Zikui', 'Longhong' and '124' were not in the same group, indicating that the content of polyphenols in pericarp and pulp was very different. 


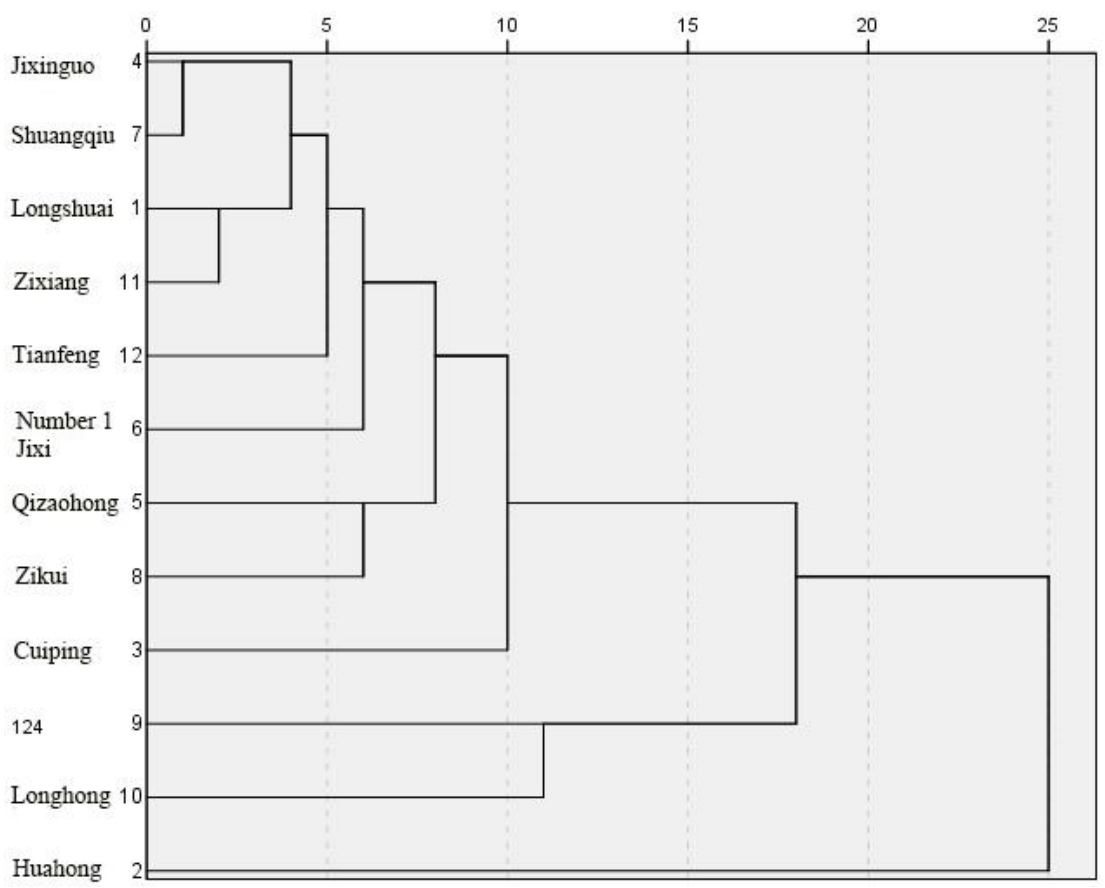

Figure 3 pericarp polyphenol clustering diagram of 12 apple landraces grown in Heilongjiang Province

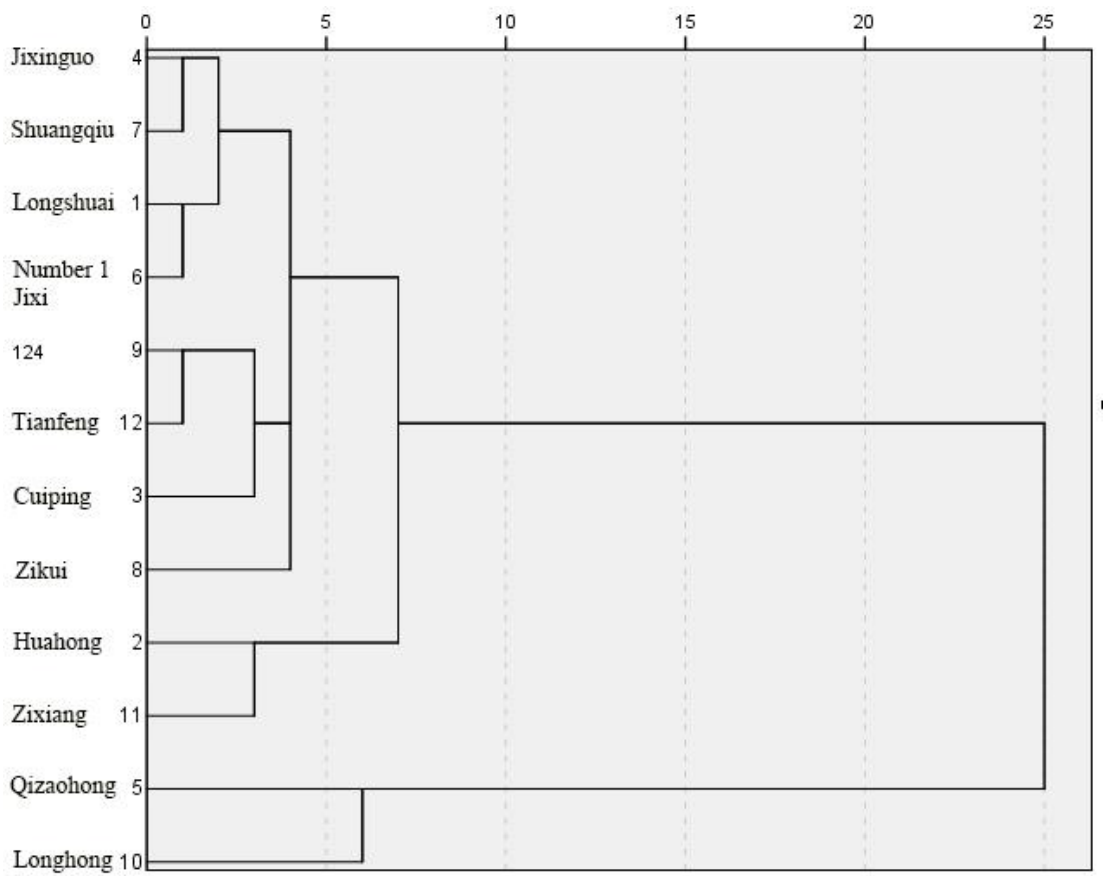

Figure 4 Pulp polyphenol clustering diagram of 12 apple landraces grown in Heilongjiang Province

\section{Discussion}

Apples have rich nutritional value and good health effects (Huang, 2019). The pericarp, pulp and seeds contain a lot of phenolic substances (Tian et al., 2011). The composition and content of different varieties, different maturity and different parts were significantly different (Ran et al., 2018; Han et al., 2018; Wei, 2019). The main substances in apple polyphenols were chlorogenic acid, epicatechin and proanthocyanidins (Nie and Sun, 2005). In this study, the main phenolic substances in pericarp of 12 apple landraces grown in Heilongiiang Province were epicatechin and catechins, and the main phenolic substances in pulp were chlorogenic acid, catechins and proanthocyanidins, which were similar to the results of previous research (Wang et al., 2003). Previous studies had found that the content of proanthocyanidin B2 was higher (Wang et al., 2017b). The results of this study 
showed that the content of proanthocyanidin B4 was higher than that of proanthocyanidin B2 and proanthocyanidin B1. At the same time, it was found that the content of quercetin galactoside and phlorizin in pericarp of different varieties were also high, and the content of quercetin galactoside in 'Longhong' was 88.38 $\mathrm{mg} / \mathrm{kg}(\mathrm{FW})$ and the content of phlorizin in 'Cuiping' was $77.41 \mathrm{mg} / \mathrm{kg}(\mathrm{FW})$. The content of gallic acid in pericarp of 'Cuiping' was $16.57 \mathrm{mg} / \mathrm{kg}(\mathrm{FW})$, and the content of protocatechuic acid in pericarp of 'Huahong' was $5.11 \mathrm{mg} / \mathrm{kg}(\mathrm{FW})$. These differential components provide a reference for the composition diversity of apple landraces grown in China.

Through the principal component analysis of apple landraces grown in Heilongjiang Province, it was found that the first four principal components played a major role in pericarp and pulp. The distribution of each component was more intuitively reflected in the two-dimensional diagram. The varieties represented by different principal components were different. Those with high polyphenolic content were all in the positive electrode and the cumulative content of eigenvalue was higher, and the content of eigenvalue near the origin was lower. In addition, gallic acid, protocatechuic acid, neochlorogenic acid and cyanidin-3-glucoside were not detected in pulp of all varieties. Some domestic scholars encountered a similar situation when studying apple landraces, which needed to be verified by adding varieties. It was also found that the polyphenolic content in pericarp of 'Huahong' was high, while the polyphenolic content in pulp of 'Qizaohong' and 'Longhong' was high, which further showed that the polyphenolic content in different parts of apple fruit were significantly different.

Through the determination of flavonoids, polyphenols and other components, some scholars at home and abroad take the content as the basis for classification of apple variety (Williams, 1982; Wang et al., 2017b). Based on the content of polyphenolic components in pericarp and pulp of apple landraces grown in Heilongjiang Province, it was found that the clustering results of polyphenols in pulp were more consistent with the results of field phenotypic identification and genetic relationship. Among the test results of pericarp, 'Longshuai' and 'Zixiang' were not consistent with field production. The female parent of 'Zixiang' was 'red Begonia' and was classified as Begonia, while the female parent of 'Longshuai' was 'Hanfu' and was classified as Big Apple. The reason may be that the pericarp was rich in polyphenols and was more vulnerable to the external environment than pulp, resulting in inaccurate determination results. Previous scholars mostly mixed pericarp and pulp to determine polyphenols, which led to no discovery of the inconsistency between clustering of pericarp and genetic relationship.

\section{Materials and Methods}

\subsection{Test materials and reagents}

Twelve test samples were collected from the Malus pumila Mill variety resource nursery of Mudanjiang Branch of Heilongjiang Academy of Agricultural Sciences from late August to early September, 2018. At the fruit ripening stage, 3 plants of each variety were selected, and 12 fruits were randomly sampled from the same height in the four directions of east, west, north and south, including 8 from Heilongjiang, 'Longshuai' Malus pumila Mill, 'Cuiping' Malus pumila Mill, 'Qizaohong' Malus pumila Mill, 'Number 1 Jixi' Malus pumila Mill, 'Shuangqiu' Malus pumila Mill, 'Zikui' Malus pumila Mill, 'Longhong' Malus pumila Mill and 'Zixiang' Malus pumila Mill, 3 from Jilin, 'Huahong' Malus pumila Mill, '124' Malus pumila Mill and 'Tianfeng' Malus pumila Mill, 1 from Inner Mongolia, 'Jixinguo' Malus pumila Mill. The samples were sent to the Key Laboratory of Biology and Genetic Improvement of Horticultural Crops (Germplasm Resources Utilization) Ministry of Agriculture of Xingcheng City, Liaoning Province in time. The pericarp and pulp were separated and stored at $-80^{\circ} \mathrm{C}$ for testing.

The standard products gallic acid, catechins, epicatechin, kaempferol, quercetin 3-galactoside, quercetin, phloem, proanthocyanidin $\mathrm{B} 1$, proanthocyanidin, quercetin-glucoside, cyanidin-rhamnoside, cyanidin-glucoside and cyanidin galactoside were purchased from Sigma company of the United States. proanthocyanidin B4, phlorizin, chlorogenic acid, cryptochlorogenic acid, neochlorogenic acid, rutin, quercetin, protocatechuic acid and cinnamic acid were purchased from Chengdu Must Bio-technology Co., Ltd of China, and the purity of standard samples was over 98\%. Chromatographic pure formic acid, acetonitrile and methanol were purchased from Fisher company of the United States, and other reagents were domestic analytical pure. 


\subsection{Instruments and equipment}

Mill-Q Academic ultrapure water system was from Mill pore company of the United States; DL-1000E ultrasonic oscillator was from Shanghai Zhixin Instrument Co., Ltd.; Thermo Genesys 10S UV-VIS ultraviolet-visible spectrophotometer was from Thermo Scientific Technology Co., Ltd. of the United States; High-speed refrigerated centrifuge 3H24RI was from Hunan Hexi Instrument Equipment Co., Ltd; RE4298 Rotary Evaporator was from Shanghai Yarong Biochemical Instrument Factory; SHZ-III circulating water vacuum pump was from Shanghai Zhixin Experimental Instrument Technology Co., Ltd; Waters QSM UPLC ultra performance liquid chromatograph was from Waters Company of the United States; HSS T3 $1.8 \mu \mathrm{m}$ reverse phase chromatography column and Oasis ${ }^{\circledR}$ HLB solid-phase extraction cartridges were also purchased from Waters Company of the United States.

\subsection{Test methods}

The polyphenolic extraction method was referred to Nie et al. (2010). Firstly, freeze and grind the apple pericarp or pulp to powder, then weigh $2.0 \mathrm{~g}$, and then extract with $5.0 \mathrm{~mL} 80 \%$ ethanol. Shake well before extraction and then ultrasound for $20 \mathrm{~min}$ was good for the dissolution of polyphenols. Centrifuge in a $10000 \mathrm{r} / \mathrm{min}$ centrifuge for $5 \mathrm{~min}$, transfer the supernatant to a $10.0 \mathrm{~mL}$ centrifuge tube, repeat the extraction with $5.0 \mathrm{~mL} 80 \%$ ethanol for once, and transfer the supernatant to a $10.0 \mathrm{~mL}$ centrifuge tube. Combine the two supernatants and the volume was fixed to $10 \mathrm{~mL}$, and remove the ethanol in $5 \mathrm{~mL}$ extract with a rotary evaporator at $40^{\circ} \mathrm{C}$. At the same time, activate Oasis HLB solid phase extraction column with $5 \mathrm{~mL}$ methanol and $5 \mathrm{~mL}$ purified water, then dissolve the polyphenols collected on the rotary evaporator with $5 \mathrm{~mL}$ methanol, filter with solid phase extraction column, and pass the filtrate through $0.22 \mu \mathrm{m}$ organic phase filter membrane, to be tested for later use.

\subsection{Data processing}

Data calculation and collation were performed with Microsoft Excel 2017, and IBM SPSS Statistics 22.0 was used for analysis of variance and cluster analysis.

\section{Authors' contributions}

LWQ and ZJR were both the executors of the experimental design and project research, and writing the first draft of the manuscript and completing the data analysis. WS and LL completed the sample collection. BHD completed the data survey. YWQ and WK participated in the experimental design. LC was the designer of the project, guiding experimental design, data analysis, manuscript writing and revision. All authors read and approved the final manuscript.

\section{Acknowledgments}

This study was jointly funded by National Natural Science Foundation of China (31701879), Special Fund for National Modern Agricultural Industrial System of China (CARS-27), the Open Fund of Key Laboratory of Biology and Genetic Improvement of Horticultural Crops (Germplasm Resources Utilization) Ministry of Agriculture of China (NYZS2018-1-2), Applied Technology Research and Development Plan Project of Heilongjiang Province (GA19B105) and Ph.D. Research Startup Fund of Yan'an University (YDBK2018-34).

\section{References}

Feng T., Yang R., Li Y.M., Feng S., and Cui P.L., 2008, Study on antioxidant activity of appke polyphenol exteracts, Shipin Yanjiu Yu Kaifa (Food Research and Development), 29(12): 189-192

Guo C.H., 2012, Research on the countermeasures of fruit industry development in Heilongjiang Province, Beifang Guoshu (Northern Fruit Tree), (4): 53-54

Han M.H., Wang C.L., Hu H.B., Wu Y., and Zhang L.L., 2018, Analysis of Compostion and Content of Polyphenols in Qingyang Red Fuji Apple, Shipin Gongye Keji (Science and Technology of Food Industry), 39(20): 244-248

Han W.F., Guo H.Y., Jia J., and Tan X.H., 2019, Research progress on polyphenols and antioxygenic property of fruits and vegetables, Baoxian Yu Jiagong (Storage and Process), 19(4): 191-194

Huang X.H., 2019, Active ingredients in apples and their health benefits, Zhongguo Ziyuan Zonghe Liyong (Comprehensive utilization of resources in China), 37(8): 179-180

Li Q.M.,Yang C.P., Li J., and Tang Z.P., 2010, Research progress in methods for extraction and isolation of plant polyphenol, Baoxian Yu Jiagong (Freshness and Processing), 10(1): 16-19

Liu J.C., Wang S.X., Jiao Z.G., Ma C.H., WANG Y.L., Guo H.X., Wang X.Y., and LI Y.J., 2005, Studies on antioxidant activities of apple polyphenol extracts in vitro, Guoshu Xuebao (Journal of Fruit Science), 22(2): 106-110

Lu Y.R., and Foo L.Y., 2000, Antioxidant and radical scavenging activities of polyphenols from apple pomace, Food Chemistry, 68(1): 81-85 https://doi.org/10.1016/S0308-8146(99)00167-3 
Nie J.Y., Lv D.G., Li J., Liu F.Z., Li H.F., Xu G.F., and Wu Y.L., 2010, Studies on Composition and Content of Flavonoids in Fruit of'Nagafu 2’Apple, Yuanyi Xuebao (Acta Horticulturae Sinica), 37(10): 1559-1566

Nie L.C., and Sun J.S., 2005, Study on the Phenolic Compound Contents in Apple of Different Cultivars, Zhongguo Shipin Xuebao (Journal of Chinese Institute of Food Science and Technology), 5(3): 118-121

Ran J.J., Zhao R.X., Ruan X.L., Liang X.H., Jiao L.X., 2018, Evaluation of Antioxidant Activity of Polyphenols from Different Varieties Apples in Vitro, Shipin Gongye Keji (Science and Technology of Food Industry), 39(23): 89-94

Song Y., Zhai H., Du Y.P., Chen F., and Wei S.W., 2006, Polyphenolic compounds and antioxidant capacity inprocessing apple cultivars, Guoshu Xuebao (JournalofFruitScience), 23(6): 793-797

Tian L.L., Liu J.L., Zheng Z.W., Niu P.F., and Guo Y.R., 2011, Research progress of compounds and physiological functions of apple polyphenol, Shipin Gongye Keji (Science and Technology of Food Industry), 32(12): 552-557

Van D.S.A., Dekker M., De,J.A., and Jongen W.M., 2001, Activity and concentration of polyphenolic antioxidants in apple:effect of cultivar,harvest year,and storage conditions, Journal of Agricultural and Food chemistry, 49(8): 3606-3613

https://doi.org/10.1021/jf001493u PMid: 11513636

Wang B., Wang X.X., Lv C.M., Meng X.J., and Song Y.H., 2011, Extraction and application of apple polyphenol from apple pomace, Shipin Gongye Keji (Food Industry Technology), 32(6): 421-424, 197

Wang D.J., Wang K., Gao Y., Zhao J.R., Liu L.J., Li L.W., and Li J., 2017a, Analysis of genetic background and characteristics of polyphenol composition and content in fruits of apple landraces grown in Hebei Province, Zhiwu Shengli Xuebao (Plant Physiology Journal), 53(8): 1515-1522

Wang D.J.,Wang K., Li J., Gao Y., Zhao J.R., Liu L.J., Gong X., and Li L.W., 2017b,Study on the content characteristics of polyphenols in 20 wild apple resources, Zhongguo Guoshu (Chinese Fruit Tree), 2017b, 4: 74-78

Wang S.X., Liu J.C., Jiao Z.G., Zhang S.N., and Yang L., 2003, Changes of polyphenols during fruit development in apples, Guoshu Xuebao (Journal of Fruit Science), 20(6): 427-431

Wei S.Q., 2019, Study on the Contents of Polyphenols in Apples with Different Maturity, Xuchang Xueyuan Xuebao (Journal Of Xuchang University), 38(2): 99-102

Williams A.H., 1982, Chemical evidence from the favonoids relevant to the classification of Malus species, Bot. J. Linn. Soc., 84(1): 31-39 https://doi.org/10.1111/j.1095-8339.1982.tb00358.x

Yan Y.F., Wang X.C., Li X.C., and Lin Z.C., 2018, Purification and comparison of polyphenols in peel, pulp and pit of different apples, Jiangxi Huagong (Jiangxi Chemical), 6: 136-137

Zhang D.Q., Tai J.X., Fu Q., 1999, Survey on research and application of Bioflavonoids, Shipin Yu Fajiao Gongye (Food and Fermentation Industries), 25(6): $52-57$

Zhang G.Z., Ji B.P., and Tian F., 2015, Composition and antioxidant activity of apple polyphenols in apple tissue zones, Shipin Gongye Keji (Food Industry Technology), 36(19): 133-137

Zhao J.R., Liu G.J., Chang R.F., Cao K., Shen F., Wu T., Wang Y., Han Z.H., and Zhang X.Z., 2015, Diversity of flesh polyphenols and their progressive dilution during fruit expansion in Malus germplasm, Scientia Horticulturae, 197: 461-469 https://doi.org/10.1016/j.scienta.2015.10.003

Zhao R.M., 2019, Biological functions and extraction methods of apple polyphenol, Shijiazhuang Zhiye Jishu Xueyuan Xuebao (Journal of Shijiazhuang University of Applied Technology), 31(2): 51-53 